\title{
The Linkage Between Bone Densitometry and Cardiovascular Disease
}

\author{
Mahmoud A. REFAEE ${ }^{\mathrm{a}, \mathrm{b}}$, Hamada R.H. AL-ABSI ${ }^{\mathrm{a}}$, Mohammad Tariqul ISLAM ${ }^{\mathrm{c}}$, \\ Mowafa HOUSEH ${ }^{\mathrm{a}}$, Zubair SHAH ${ }^{\mathrm{a}}$, M.Sohel RAHMAN ${ }^{\mathrm{d}}$ and Tanvir ALAM ${ }^{\mathrm{a}, 1}$ \\ ${ }^{a}$ College of Science and Engineering, Hamad Bin Khalifa University, Doha, Qatar \\ ${ }^{\mathrm{b}}$ Hamad Medical Corporation, Doha, Qatar \\ ${ }^{c}$ Computer Science Department, Southern Connecticut State University, New Haven, \\ CT 06515 , USA \\ ${ }^{\mathrm{d}}$ Department of Computer Science \& Engineering, Bangladesh University of \\ Engineering and Technology, Dhaka, Bangladesh
}

\begin{abstract}
Dual-energy X-ray absorptiometry (DXA) has been traditionally used to assess body composition covering bone, fat and muscle content. Cardiovascular disease (CVD) has deleterious effects on bone health and fat composition. Therefore, early detection of bone health, fat and muscle composition would help to anticipate a proper diagnosis and treatment plan for CVD patients. In this study, we leveraged machine learning (ML)-based models to predict CVD using DXA, demonstrating that it can be considered an innovative approach for early detection of CVD. We leveraged state-of-the-art ML models to classify the CVD group from non-CVD group. The proposed logistic regression-based model achieved nearly $80 \%$ accuracy. Overall, the bone mineral density, fat content, muscle mass and bone surface area measurements were elevated in the CVD group compared to non-CVD group. Ablation study revealed a more successful discriminatory power of fat content and bone mineral density than muscle mass and bone areas. To the best of our knowledge, this work is the first ML model to reveal the association between DXA measurements and CVD in the Qatari population. We believe this study will open new avenues of introducing DXA in creating the diagnosis and treatment plan of cardiovascular diseases.
\end{abstract}

Keywords. Cardiovascular disease, Dual-energy X-ray absorptiometry (DXA), Bone densitometry, Qatar Biobank (QBB)

\section{Introduction}

Dual X-ray Absorptiometry (DXA) is a fast and non-invasive approach to measure the body composition in terms of mass, fat and bone composition. Using DXA, the entire body can be scanned to calculate the composition of bone mass and soft tissue. DXA machine can also reveal results of fat content and lean muscle of the body when a fullbody scanning is performed [1]. DXA is a standard method for evaluating bone mass and body composition for several reasons. First, the two X-ray attenuations from the DXA machine that pass through the body can measure the mass using the simple algebra and physical properties of materials. It can also measure the regional body compositions by

${ }^{1}$ Corresponding Author, Tanvir Alam, College of Science and Engineering, Hamad Bin Khalifa University, Doha, Qatar; E-mail: talam@hbku.edu.qa. 
subdividing the whole body into well-defined body cut lines. Overall, it is a precise and stable measurement technique that has been used in clinics for several years. By using DXA Phantoms, it is quite simple to also verify measurement stability (nearly $0.5 \%$ variation) for body composition [1]. Recently, Hologic Inc. has started to use DXA for the prediction of cardiovascular diseases, including coronary heart disease, stroke, etc. This process is already approved by the FDA [2]. Lastly, this method can facilitate the discovery of comorbidities and mortality in cohorts from multiple ethnicities, as well.

According to the WHO, cardiovascular disease (CVD) is considered as the primary cause of fatality worldwide. In its 2018 report, The World Health Organization (WHO) stated that in 2016, non-communicable diseases (NCD) account for more than $70 \%$ of the global death. Out of this, CVDs contributed to over $44 \%$ of total NCD-related mortality. In the Gulf Cooperation Council Countries, CVD is the leading cause of fatality [3]. The Ministry of Public Health, Qatar has reported CVD as the leading cause of fatality in Qatar, with CVD now being considered as an economic burden in Qatar's healthcare sector. Many studies exist focusing on the early detection of CVD using machine learning (ML)-based approaches [4]. To our knowledge, no study has been published focusing on the early detection of CVD using DXA measurements in Qatar based on ML techniques. The objective of this study is to leverage DXA measurements to determine if they are effective in the diagnosis of CVD. We formulated this problem in the machine learning classification framework to classify the CVD group from the non-CVD group. Apart from diagnosing CVD, the proposed ML model will also help us to associate different metrics from body compositions to CVD in the Qatari population.

\section{Materials and Methods}

\subsection{Data Collection from $Q B B$}

We collected a deidentified dataset from Qatar Biobank (QBB) for a CVD cohort of 250 participants. We also had a control (non-CVD) group of 250 cases that were free from CVD and other related diseases like diabetes, obesity etc. In the CVD group, most participants were diagnosed with hypertension (180 out of 250 , nearly $72 \%$ ). Other participants from the CVD group had abnormal heartbeats, angina, and revascularization.

\subsection{Data Description and Preprocessing}

There were 182 different measurements from DXA in the dataset. DXA machines primarily capture four types of measurements from different body parts: namely, (i) bone mineral density (BMD), (ii) lean mass, (iii) fat content, and (iv) bone area measurements. A few metrics from each category are described in Table 1.

Table 1: List of some measurements from the DXA dataset

\begin{tabular}{ll}
\hline Data Type & \multicolumn{1}{c}{ Description } \\
\hline BMD & $\begin{array}{l}\text { Age-matched Z-score in the body area, Young adult T-Score in total body, BMD } \\
\left(\mathrm{g} / \mathrm{cm}^{2}\right) \text { in total body, spine, etc. }\end{array}$ \\
Lean mass & Tissue lean mass $(\mathrm{g})$ in leg, android, trunk, gynoid, etc. \\
Fat content & Tissue fat mass $(\mathrm{g})$ in the trunk, arm, android, gynoid, total body etc. \\
Bone Area & Bone area $\left(\mathrm{cm}^{2}\right)$ in total body, legs, lumbar spine, etc. \\
\hline
\end{tabular}


Features with over $30 \%$ missing values were removed. For the remaining features, the missing values were replaced with the corresponding feature-mean. Data from both the CVD group and control group were normalized applying min-max normalization.

\subsection{Machine Learning Model Development and Evaluation}

We developed multiple ML models to distinguish the CVD group from the non-CVD group. We used four different ML algorithms: Multi-layer Perceptron (MLP), Decision Tree (DT), Random Forest (RF), and Logistic Regression (LR). We applied five-fold cross validation to evaluate the model. We considered multiple performance evaluation metrics such as: (i) Accuracy, (ii) Sensitivity, (iii) Specificity, and (iv) Matthews' Correlation Coefficient (MCC) to evaluate the performance of the proposed ML models.

\section{Results and Discussions}

In the CVD group, $54.80 \%$ participants were male and $45.2 \%$ were female, whereas in the control group had an even distribution of $50 \%$ between male and female participants). All the participants included in this study were adult Qatari nationals. The weight (72.2:64.2 in CVD:control) and BMI (26.12:23.2 in CVD:control) were relatively greater in the CVD group compared to the non-CVD group. In addition to considering the full DXA measurements in the ML model as features, an ablation study was conducted to measure the effectiveness of different types of DXA measurements by feeding them into ML models as different feature groups. Table 2 compares the performance of the ML models. Evidently, the LR-based model achieved the accuracy of $74 \%$ for BMD, $70 \%$ for lean mass, $76 \%$ for fat contents and $70.8 \%$ for bone area measurements. Considering all DXA measurements (182 features), the LR-based model achieved the highest accuracy of $77.4 \%$.

Table 2 Machine Learning models performance based on ablation study

\begin{tabular}{|c|c|c|c|c|c|}
\hline Feature & Model & Accuracy & Sensitivity & Specificity & MCC \\
\hline BMD & DT & 0.718 & 0.648 & 0.798 & 0.455 \\
\hline \multirow[t]{3}{*}{ (85 features) } & MLP & 0.688 & 0.746 & 0.642 & 0.390 \\
\hline & $\mathrm{RF}$ & 0.690 & 0.626 & 0.756 & 0.386 \\
\hline & LR & 0.740 & 0.709 & 0.775 & 0.487 \\
\hline Lean mass & DT & 0.662 & 0.643 & 0.679 & 0.326 \\
\hline \multirow[t]{3}{*}{ (7 features) } & MLP & 0.592 & 0.437 & 0.730 & 0.171 \\
\hline & $\mathrm{RF}$ & 0.672 & 0.654 & 0.689 & 0.344 \\
\hline & LR & 0.700 & 0.642 & 0.757 & 0.402 \\
\hline \multirow{4}{*}{$\begin{array}{l}\text { Fat content } \\
\text { (15 features) }\end{array}$} & DT & 0.722 & 0.655 & 0.787 & 0.449 \\
\hline & MLP & 0.724 & 0.739 & 0.715 & 0.458 \\
\hline & $\mathrm{RF}$ & 0.744 & 0.679 & 0.801 & 0.486 \\
\hline & LR & 0.764 & 0.722 & 0.806 & 0.551 \\
\hline \multirow{4}{*}{$\begin{array}{l}\text { Bone area } \\
\text { measurements } \\
\text { ( } 75 \text { features) }\end{array}$} & DT & 0.646 & 0.600 & 0.702 & 0.301 \\
\hline & MLP & 0.636 & 0.723 & 0.572 & 0.296 \\
\hline & $\mathrm{RF}$ & 0.642 & 0.626 & 0.657 & 0.281 \\
\hline & LR & 0.708 & 0.670 & 0.756 & 0.424 \\
\hline \multirow{4}{*}{$\begin{array}{l}\text { All features } \\
(182)\end{array}$} & DT & 0.700 & 0.625 & 0.779 & 0.413 \\
\hline & MLP & 0.678 & 0.677 & 0.681 & 0.363 \\
\hline & $\mathrm{RF}$ & 0.740 & 0.686 & 0.796 & 0.491 \\
\hline & LR & 0.774 & 0.757 & 0.797 & 0.556 \\
\hline
\end{tabular}


Our results demonstrated better bone health for the CVD group. The young adult Tscore for lumbar spines (L1, L2, L4) were greater in the CVD group compared to nonCVD group. The Z-score for L2-L4 was -0.282 : -0.57 in CVD vs. control group. Additionally, bone mass total was 2,510.45 in the CVD group vs. 2,416.17 in the control group. We also observed higher total lean mass (43,643.57: 40,614.88 in CVD:control) in CVD compared to the control group. Another prospective study based on the Greek general population recruited 3,042 adults without pre-existing CVD and the baseline skeletal muscle mass revealed an inverse association with ten-year CVD incidence [5]. Our study also observed higher fat content in the body composition of the CVD group (total fat mass=25908.57: 20962.12 in CVD: control). As mentioned earlier, the studied CVD group had higher BMI levels than the non-CVD group, and a higher weight elevated the fat level in the CVD group. In summary, most of the bone mineral density, fat content, muscle mass and bone surface area related measurements were greater in the studied CVD group compared to non-CVD group. The details of the DXA measurements can be found at: https://github.com/tanviralambd/CVD_DXA.

This study has some limitations worth mentioning. We worked with a relatively smaller sized cohort and we did not consider lifestyle or socio-demographic information of the participants in the model. Integrating such valuable information would add more insights into our study.

\section{Conclusion}

Although the FDA approved the use of DXA for diagnosing CVD recently, which measurements and what formula should be used in predicting and expanding the usage of DXA to CVD conditions still needs to be determined. The current study observes that machine learning can help in the selection of DXA measurements, which can potentially be included in the final report for more convenient clinical practice. In the future, we will evaluate our proposed approach in the CVD group with more advanced stages like ischemic heart attack and revascularization.

\section{References}

[1] Shepherd JA, Ng BK, Sommer MJ, Heymsfield SB. Body composition by DXA. Bone. 2017;104:1015 .

[2] Horizon DXA System [Internet]. [cited 2021 Aug 27]. Available from: https://www.hologic.com/hologic-products/skeletal-solutions/horizon-dxa-system.

[3] Al-Absi HRH, Refaee MA, Nazeemudeen A, Househ M, Shah Z, Alam T. Cardiovascular Diseases in Qatar: Smoking, Food Habits and Physical Activities Perspectives. Stud Health Technol Inform. 2020;272:465-9.

[4] Al-Absi HRH, Refaee MA, Rehman AU, Islam MT, Belhaouari SB, Alam T. Risk Factors and Comorbidities Associated to Cardiovascular Disease in Qatar: A Machine Learning Based Case-Control Study [Internet]. IEEE Access. 2021. p. 29929-41. Available from: http://dx.doi.org/10.1109/access.2021.3059469

[5] Tyrovolas S, Panagiotakos D, Georgousopoulou E, Chrysohoou C, Tousoulis D, Haro JM, et al. Skeletal muscle mass in relation to 10 year cardiovascular disease incidence among middle aged and older adults: the ATTICA study. J Epidemiol Community Health. 2020;74:26-31. 